\title{
A "Real-World" Chart Review Study of Monotherapy and Adjunctive Vortioxetine in Clinical Practice
}

\author{
Sagar Karia ${ }^{1}$, Ruksheda Syeda ${ }^{2}$, Avinash De Sousa ${ }^{3}$
}

\begin{abstract}
Background: Vortioxetine is a novel antidepressant drug that proved effective in the treatment of major depressive disorder (MDD) and anxiety disorder. It is a unique antidepressant having a multimodal serotonergic mechanism of action and also has important effects on other neurotransmitters implicated in the neurobiology of depression. Keeping in mind the sparse literature on vortioxetine from India, this real-world chart review was conducted to assess its utility and effectiveness.

Materials and methods: This is a multicentric retrospective chart analysis of all patients who were prescribed vortioxetine in outpatient psychiatric clinics set in urban areas. Patients prescribed the drug between January 1,2019, and December 31, 2019, were included in the study. The chart review included details regarding the reason for starting vortioxetine, dosage, side effects observed, duration, and adjunctive medications that the patient had. Descriptive statistics were used in the analysis and the data were presented.

Results: The chart review had data of 131 patients. Of these, 85 patients are still maintained on vortioxetine. Majority of the subjects suffered from a MDD. Other indications for the use of vortioxetine included anxiety disorders, obsessive-compulsive disorders, and borderline personality disorder. Only 12 patients suffered side effects which were minor. Majority of the patients reported good improvement with the drug and cost of the drug was a major deterrent to continuing long-term treatment with the drug.

Conclusions: Vortioxetine was found to be efficacious with minimal side effects. It may improve cognition in depression and has a lower incidence of treatment-emergent sexual dysfunction. Further data from diverse patient populations are needed before we comment on its effectiveness and safety.

Keywords: Cognition, Depression, Side effects, Vortioxetine.

Indian Journal of Private Psychiatry (2020): 10.5005/jp-journals-10067-0059
\end{abstract}

\section{INTRODUCTION}

Major depressive disorder (MDD) is a chronic, lifelong psychiatric disorder that has a substantial impact on public health, quality of life, and socio-occupational functioning. ${ }^{1}$ Many antidepressants of different classes are available for the management of MDD but yet many patients fail to respond completely to treatment and relapse rates are high. ${ }^{2}$ Vortioxetine is one of the newer antidepressants belonging to the class of bis-arylsulfanylamines. ${ }^{3}$ First approved in 2013 by the US Food and Drug Administration (FDA) for the treatment of MDD in adults, the European Medicines Agency (EMA) next approved vortioxetine in the same year. It is available for oral administration and in strengths of 5,10 , and $20 \mathrm{mg}$ tablets. ${ }^{4} \mathrm{It}$ has a novel mechanism of action by inhibiting the serotonin transporter (SERT) and antagonizes several serotonin receptors $\left(5-\mathrm{HT}_{3}, 5-\mathrm{HT}_{7}\right.$, and $\left.5-\mathrm{HT}_{1 \mathrm{D}}\right){ }^{5}$ It also acts as a partial agonist on $5-\mathrm{HT}_{1 \mathrm{~B}}$ receptors and as a full agonist on $5-\mathrm{HT}_{1 \mathrm{~A}}$ receptors. It is this action that may also explain its effects on anxiety in addition to mood and depression. ${ }^{6}$ The molecule has been introduced in India in October 2018 and has been used in the management of MDD. There are some reports on its effectiveness in improving cognition in MDD, and its utility in generalized anxiety disorder ${ }^{8}$ and obsessive compulsive disorder. ${ }^{9}$ There is a scarcity of Indian data on the use of vortioxetine hence we present this retrospective chart review of its utility and effectiveness in Indian patients in a real-world clinical private practice setting.

\section{Materials and Methods}

The charts of 131 adult outpatients (72 men, 59 women) with a DSM $-5^{10}$ diagnosis of MDD and other psychiatric disorders attending the psychiatry outpatient department of a medical

\footnotetext{
1,3 Department of Psychiatry, Lokmanya Tilak Municipal Medical College, Mumbai, Maharashtra, India

${ }^{2}$ Trellis Clinic - Private Practice, Mumbai, Maharashtra, India

Corresponding Author: Avinash De Sousa, Department of Psychiatry, Lokmanya Tilak Municipal Medical College, Mumbai, Maharashtra, India, Phone: +91 9820696828, e-mail: avinashdes888@gmail.com
}

How to cite this article: Karia S, Syeda R, De Sousa A. A "Real-World" Chart Review Study of Monotherapy and Adjunctive Vortioxetine in Clinical Practice. Ind J Priv Psychiatry 2020;14(2):92-94.

Source of support: Nil

Conflict of interest: None

college and two private psychiatric outpatient clinics between January 1, 2019, and December 31, 2019, were reviewed retrospectively. As this was a retrospective chart review and did not involve a direct interview with patients, ethics committee approval was not taken but it was passed in the departmental review meeting. All the psychiatrists had over 10 years of clinical experience and made diagnoses of the patients. Several enrolled patients took low dosages of benzodiazepines (BDZs), mood stabilizers, and other antidepressants. There were no fixed inclusion and exclusion criteria. Data were collected from the records that consultants had with them and those patients who were on vortioxetine were included in this chart review. No rating scales were used in the assessment and improvement and side effects were clinically assessed along with patient input. Any incident adverse events were evaluated through chart review at every follow-up visit and at the end of the treatment 
period. Vortioxetine dosage was flexible and adjusted clinically based on the needs of the patient. Details regarding the reason for starting the drug, starting dose, maximum dose attained and maintenance dose, side effects observed, and duration for which they took were recorded by using a semistructured questionnaire. All patients in the study were given the option of taking vortioxetine for their treatment after explaining the benefits and cost of the molecule. There was no randomization. All patients were informed that their data would be used in a chart review with anonymity and they had agreed to the same. The study was discussed in a department review meeting of a tertiary general hospital. Descriptive statistics like frequency and percentages were used in the analysis.

\section{Results}

One hundred and thirty-one patients were included in the chart review. The mean age of our sample was $38.09 \pm 14.63$ years (range, 14-82 years). The sample consisted of 72 females ( $55 \%$ ) and 59 males (45\%). Table 1 depicts the diagnosis profile of the sample. Majority of them were given vortioxetine for treatment of depression ( $n-110$, $83.9 \%)$. In this depression group, there was clinical heterogeneity as $20.6 \%(n=27)$ had a specifier of anxious distress and $10.6 \%(n=14)$ had comorbid borderline personality disorder. Sixty-four (48.86\%) of them were on monotherapy while sixty-seven (51.14\%) were given vortioxetine as an adjunctive medication. Twenty (15.27\%) patients had been started on psychotropics for the first time while others had shown poor response to previous pharmacotherapy. Twenty (15.27\%) of these patients had comorbid diabetes mellitus, twenty-five (19.08\%) had hypertension, and six (4.59\%) had thyroid

Table 1: Diagnosis of patients in whom vortioxetine prescribed

\begin{tabular}{lll}
\hline Diagnosis of the patients & $\begin{array}{l}\text { Number of patients (\%) } \\
(N=131)\end{array}$ \\
\hline 1 & $\begin{array}{l}\text { Major depressive disorder } \\
\text { (MDD) and related disorders }\end{array}$ & $110(83.9 \%)$ \\
2 & Bipolar mood disorder & $5(3.8 \%)$ \\
3 & Anxiety disorder & $6(4.6 \%)$ \\
4 & Eating disorder & $1(0.8 \%)$ \\
5 & Somatic symptom disorder & $2(1.5 \%)$ \\
6 & Obsessive compulsive disorder & $3(2.3 \%)$ \\
7 & (OCD) & $1(0.8 \%)$ \\
8 & Mild cognitive disorder & $1(0.8 \%)$ \\
9 & Dementia & $2(1.5 \%)$ \\
Diagnosis split in MDD and related & Number of patients (\%) \\
disorders category & $(N=110)$ \\
1 & MDD & $62(56.4 \%)$ \\
2 & MDD with anxious distress & $22(20.0 \%)$ \\
3 & MDD with psychotic features & $4(3.6 \%)$ \\
4 & MDD and borderline personali- & $12(10.9 \%)$ \\
5 & ty disorder & $3(2.7 \%)$ \\
6 & MDD and OCD & $5(4.6 \%)$ \\
7 & MDD and alcohol use disorder \\
\hline & MDD and cannabis use disorder & $1(0.9 \%)$ \\
\hline & & $1(0.9 \%)$ \\
\hline
\end{tabular}

disorders. Ninety-seven (74.05\%) of patients were started on vortioxetine due to sadness of mood, eleven (8.39\%) were started on the drug for anxiety symptoms, and seven (5.35\%) were started for passive suicidal ideation. Ninety $(68.7 \%)$ were started at a dose of $5 \mathrm{mg}$ dose and forty-one (31.3\%) were started at $10 \mathrm{mg}$. The maximum dose given was $40 \mathrm{mg}(n=4)$ and one patient had received $30 \mathrm{mg}$. The mean duration for which patients were on vortioxetine was $4.37 \pm 3.14$ months $(N=109$, as other patients either did not take vortioxetine or were lost to follow-up so were not included in the calculation.) Of these 131 patients, 85 (64.9\%) are still on vortioxetine. Reasons for stopping have been depicted in Table 2. Seventy-two patients (54.96\%) claimed much improvement after starting vortioxetine but their main concern of patients was its cost. When we evaluated the side effects caused, 118 (90.08\%) of them did not experience any side effects. Five patients had gastric side effects, one had a headache, and six of them experience a switch to mania for which the medication had to be withdrawn (Table 2). Also, the side effects were experienced by the patients in the initial 7-10 days of starting vortioxetine, and these side effects improved after this period. Only those who had intolerable side effects did not continue the tablet or those who switched to mania were not continued on it.

\section{Discussion}

Our chart review has shown that vortioxetine was effective in the management of MDD both as monotherapy and as an adjunctive medication with minimal side effects. As vortioxetine has been approved for the treatment of depression, we had majority of our sample population diagnosed with MDD. Other indications mentioned in which it was used were based on clinicians' judgment, so they were very less in number. Vortioxetine, as mentioned, has multimodal activity and it increases levels of serotonin, norepinephrine (noradrenaline), dopamine, acetylcholine, and histamine in the ventral hippocampus and the medial prefrontal cortex, thereby explaining its role in depression and anxiety disorders. ${ }^{11}$ Vortioxetine has been shown to improve processing speed, executive function, and attention, when compared with placebo and this improvement in cognition is a direct effect rather than reduction in depression. ${ }^{12}$ The advantage of vortioxetine is that it undergoes metabolism by several CYP450 enzymes (including

Table 2: Reasons given by patients for stopping vortioxetine and side effects

\begin{tabular}{lll}
\hline Sr. No & Reason for stopping & Number of patients $(\%)(N=46)$ \\
\hline 1 & Lost to follow-up & $17(36.9 \%)$ \\
2 & Due to side effects & $3(6.5 \%)$ \\
3 & Switch to mania & $5(10.9 \%)$ \\
4 & Found too expensive & $10(21.7 \%)$ \\
5 & No enough improve- & $6(13.1 \%)$ \\
& ment noted & \\
6 & Felt completely better & $5(10.9 \%)$ \\
Sr. No & Side effects & Number of patients (\%) $N=131$ \\
1 & None & $119(90.8 \%)$ \\
2 & Gastric side effects like & $5(3.8 \%)$ \\
3 & nausea & $1(0.8 \%)$ \\
4 & Headache & $6(4.6 \%)$ \\
\hline
\end{tabular}


CYP2D6, CYP3A4/5, CYP2C9, CYP2C19, CYP2A6, CYP2C8, and CYP2B6) with subsequent glucuronic acid conjugation, CYP2D6 being the primary enzyme. The metabolism is not affected much by gender, race, renal impairment, and hepatic impairment, and hence, the drug can be used safely in all age-groups and genders. ${ }^{13}$ The partial agonist effect of vortioxetine on the postsynaptic $5 \mathrm{HT}_{1 \mathrm{~B}}$ heteroreceptors located in GABAergic interneurons may increase the release of glutamate in the hippocampus and prefrontal cortex, which may further contribute to an antidepressant effect. ${ }^{14}$ It also increases the clinical effectiveness of vortioxetine in treating cognitive symptoms, commonly seen in patients who give a suboptimal or inadequate response to other antidepressants and this may result in complete remission of depression in everyday clinical practice. ${ }^{15}$

The most common side effect noted in studies has been mild and transient nausea occurring in $20-30 \%$ of patients. ${ }^{16}$ Other side effects like dry mouth, dizziness, insomnia-related problems, sexual dysfunction, fatigue, and hyperhidrosis appear less frequent with vortioxetine across a daily dosage of 5-20 mg compared to venlafaxine or duloxetine. ${ }^{17}$ Switching to mania after starting vortioxetine needs to be further studied and in our chart review, six patients had a mania switch of which only one was a known case of bipolar disorder. There have been very few case reports in the literature showing manic and hypomanic switch on vortioxetine. ${ }^{18,19}$ None of our patients complained of sexual dysfunction after starting vortioxetine. Our findings are based on clinical observation and not on randomization and blinding, so this was a major limitation of our study. Further studies and randomized controlled clinical trials with head-on comparison with other antidepressants are warranted.

\section{References}

1. Lépine JP, Briley $M$. The increasing burden of depression. Neuropsychiatr Dis Treat 2011;7(Suppl 1):3-7. DOI: 10.2147/NDT. S19617.

2. Greenberg PE, Fournier AA, Sisitsky T, et al. The economic burden of adults with major depressive disorder in the United States (2005 and 2010). J Clin Psychiatry 2015;76(2):155-162. DOI: 10.4088/ JCP.14m09298.

3. Sanchez C, Asin KE, Artigas F. Vortioxetine, a novel antidepressant with multimodal activity: review of preclinical and clinical data. Pharmacol Therapeut 2015;145:43-57. DOI: 10.1016/j. pharmthera.2014.07.001.

4. Deardorff WJ, Grossberg GT. A review of the clinical efficacy, safety and tolerability of the antidepressants vilazodone, levomilnacipran and vortioxetine. Exp Opin Pharmacother 2014;15(17):2525-2542. DOI: $10.1517 / 14656566.2014 .960842$.

5. Stahl SM. Modes and nodes explain the mechanism of action of vortioxetine, a multimodal agent (MMA): enhancing serotonin release by combining serotonin $(5 \mathrm{HT})$ transporter inhibition with actions at 5HT receptors (5HT1A, 5HT1B, 5HT1D, 5HT7 receptors). CNS Spectr 2015;20(2):93-97. DOI: 10.1017/S1092852915000139.

6. Katona $\mathrm{CL}$, Katona CP. New generation multi-modal antidepressants: focus on vortioxetine for major depressive disorder. Neuropsychiatr Dis Treat 2014;10:349-354. DOI: 10.2147/NDT.S39544.

7. Gibb A, Deeks ED. Vortioxetine: first global approval. Drugs 2014;74(1):135-145. DOI: 10.1007/s40265-013-0161-9.

8. Pae CU, Wang SM, Han C, et al. Vortioxetine, a multimodal antidepressant for generalized anxiety disorder: a systematic review and meta-analysis. J Psychiatr Res 2015;64:88-98. DOI: 10.1016/j. jpsychires.2015.02.017.

9. De Berardis D, Olivieri L, Nappi F, et al. Vortioxetine and aripiprazole combination in treatment-resistant obsessive-compulsive disorder: a case report. J Clin Psychopharmacol 2017;37(6):732-734. DOI: 10.1097/ JCP.0000000000000801.

10. American Psychiatric Association. Diagnostic and statistical manual for the classification of psychiatric disorders. 5th ed. New York: American Psychiatric Publishing; 2013.

11. Pehrson AL, Leiser SC, Gulinello M, et al. Treatment of cognitive dysfunction in major depressive disorder-a review of the preclinical evidence for efficacy of selective serotonin reuptake inhibitors, serotonin-norepinephrine reuptake inhibitors and the multimodal-acting antidepressant vortioxetine. Eur J Pharmacol 2015;753:19-31. DOI: 10.1016/j.ejphar.2014.07.044.

12. McIntyre RS, Lophaven S, Olsen CK. A randomized, double-blind, placebo-controlled study of vortioxetine on cognitive function in depressed adults. Int J Neuropsychopharmacol 2014;17(10):15571567. DOI: $10.1017 /$ S1461145714000546.

13. Chen G, Højer AM, Areberg J, et al. Vortioxetine: clinical pharmacokinetics and drug interactions. Clin Pharmacokinet 2018;57(6):673-686. DOI: 10.1007/s40262-017-0612-7.

14. Riga MS, Sánchez C, Celada P, Artigas F. Involvement of 5-HT3 receptors in the action of vortioxetine in rat brain: focus on glutamatergic and GABAergic neurotransmission. Neuropharmacology 2016;108:7381. DOI: 10.1016/j.neuropharm.2016.04.023.

15. Mclntyre RS, Harrison J, Loft $\mathrm{H}$, et al. The effects of vortioxetine on cognitive function in patients with major depressive disorder: a meta-analysis of three randomized controlled trials. Int J Neuropsychopharmacol 2016;19(10):pyw055. DOI: 10.1093/ijnp/ pyw055.

16. Connolly KR, Thase ME. Vortioxetine: a new treatment for major depressive disorder. Exp Opin Pharmacother 2016;17(3):421-431. DOI: 10.1517/14656566.2016.1133588.

17. Schatzberg AF, Blier $P$, Culpepper $L$, et al. An overview of vortioxetine. J Clin Psychiatry 2014;75(12):1411-1418. DOI: 10.4088/ JCP.14027ah1.

18. Sobreira G, Oliveira J, Brissos S. Vortioxetine-induced manic mood switch in patient with previously unknown bipolar disorder. Braz J Psychiatry 2017;39(1):86-88. DOI: 10.1590/1516-4446-2016-2113.

19. Pirdoğan Aydın E, Dalkıran M, Özer ÖA, et al. Hypomanic switch during vortioxetine treatment: a case report. Psychiatr Clin Psychopharmacol 2019;29(1):114-116. DOI: 10.1080/24750573.2018.1435602. 